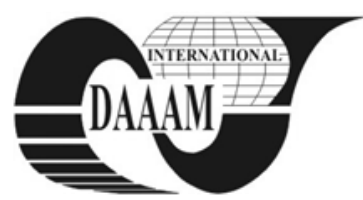

\title{
STUDY ON EVALUATING ANTIOXIDANT CAPACITY AND BIOLOGICALLY ACTIVE COMPOUNDS FROM BASIL
}

\author{
DABIJA, A[driana]; CONSTANTINESCU POP, C[ristina] G[abriela]; BUCULEI, A[melia] \& REBENCIUC, \\ I[oana]
}

\begin{abstract}
The study aims to determine the content of flavonoids, polyphenolcarboxylic acids, total polyphenols and antioxidant capacity of dried basil used in food. Investigations were performed on extracts obtained from hot and cold water solutions, methanol, ethanol and various mixtures of solvents. Data analysis show that bioactive substances are well represented in basil, which may explain the remarkable antioxidant capacity observed and the concern of introducing this particular plant when creating foods with biological potential.
\end{abstract}

Key words: spices, extraction, bioflavonoids, spectrophotometry

\section{INTRODUCTION}

Oxidative stress, which is caused by free radicals, is considered the cause of many diseases in our civilization: diabetes, cardiovascular disease, various cancers and aging processes. One of the ways to prevent the above mentioned pathologies is a proper infusion of antioxidants (Kaefer \& Milner, 2008).

In recent years, antioxidant substances from plants are of interest to researchers, producers and consumers. Good sources of antioxidants are fresh fruit and vegetables, whole grains, due to the intake of vitamins, bioflavonoids, and components with antiradical potential. Many spices are the sources of phenolic compounds with an antioxidant capacity superior to that of fruit and cereals (Lu, Mei et al., 2010). Concentrated in just a few grams of material, they may represent the simplest way to increase the content of phenolic compounds and antioxidant capacity of a daily diet, with potential health benefits (Suhaj, M., 2006). Among the factors leading to differences in composition and antioxidant activity of spices used for cooking, may be mentioned: the genetic factors, the degree of maturity of plants, cultivation techniques, post-harvest handling, storage conditions, methods applied for conditioning, processing techniques adopted etc. (Cai, Y. et al., 2004; Shan et al., 2005).

Basil (Ocimum basilicum), one of the most popular plants, a 'bridge' between medicine, food and tradition, is an annual plant of the Labiatae family, with a taste/flavour of sweet-spicy, spicy-hot. It has various uses, both culinary for flavouring and seasoning (salads, sauces, fish dishes, Mediterranean cuisine and Asian cuisine especially) and in medicine (being used as a remedy for many diseases: intestinal colic, intestinal bloating, vomiting, flu, cold, dry bronchitis, acute and chronic headaches, stomach ulcer, urinary infections, anorexia, diarrhoea, colitis fermentation).

The purpose of the study is to assess the antioxidant capacity and phenolic compounds present in basil using different extraction methods described in literature.

\section{MATERIALS AND METHODS}

For comparison purposes, a qualitative and quantitative chemical study was performed on extracts from two samples of basil: a sample consisting of basil for seasoning (sample K), and a sample consisting of basil processed for medicinal purposes (tea - sample M).

Due to the fact that the extraction technique decisively influences the results and gives a more complete perspective on the content of the analyzed compounds, extraction conditions were varied, working both at room temperature and reflux temperature, with several types of extraction solvents, met and recommended by the literature: - water, cold $\left(\mathrm{S}_{1 \mathrm{R}}\right)$ and hot $\left(\mathrm{S}_{1 \mathrm{C}}\right)$; - methanol solution $(50: 50, \mathrm{v} / \mathrm{v})$, cold $\left(\mathrm{S}_{2 \mathrm{R}}\right)$ and hot $\left(\mathrm{S}_{2 \mathrm{C}}\right)$; with ethanol at $96^{\circ}$, cold $\left(\mathrm{S}_{3 \mathrm{R}}\right)$ and hot $\left(\mathrm{S}_{3 \mathrm{C}}\right)$; - with a mixture of methanol-water-acetic acid (90:9:1, v/v/v), cold (S4); - with a mixture of methanol-acetone-water-formic acid (40:40:19, 9:0, $1, \mathrm{v} / \mathrm{v} / \mathrm{v} / \mathrm{v})$, cold $\left(\mathrm{S}_{5}\right)$.

Thus, eight samples for each type of plant (spice medicinal plant) were analyzed: five extracted hot and three extracted cold. They were obtained by leaching the plant material with an appropriate solvent. For this, each $2 \mathrm{~g}$ of dried aerial part of plant were extracted with $20 \mathrm{ml}$ solvent (ratio of plant product/solvent of 1/10), hot (reflux temperature) and cold (room temperature). Extraction solutions were filtered and were analyzed in terms of total flavone content (expressed in rutoside) and total polyphenols (as gallic acid) and antiradical capacity.

For qualitative analysis of active principles from the obtained extracts the method of thin layer chromatography (TLC) was used. Reference solutions that were used are rutoside - chlorogenic acid - caffeic acid (E1) and rosmarinic acid (E2). The quantitative chemical study sought to quantify, using spectrophotometry, the biologically active compounds (flavonoids polyphenolcarboxylic acids, total polyphenols) of the analyzed samples, knowing that spicy aromatic plants contribute substantially to the shaping of antioxidant activity. A spectrophotometric method was used in visible and ultraviolet.

Total flavone content was expressed in $\mathrm{g}$ rutoside $/ 100 \mathrm{~g}$ dried plant. Content of polyphenolcarboxylic acids was expressed as g rozmarinic acid/100d dried plant product. Total polyphenol content was expressed as gallic acid equivalents (GAE). Determination of antiradical capacity was performed by measuring the ability of neutralizing radical 2,2-diphenyl-1picrilhidrazil (DPPH) and transforming it in a reduced form by the plant extracts examined. Results were expressed as percentage inhibition of DPPH site.

To obtain these determination were used: spectrophotometers UV-VIS CARY 50, CECIL 2020, CINTRA 101, V-550 Jasco, applicator: CAMAG LINOMAT IV; G60F254 Merck silica gel HPTLC plates $(100 \times 100,200 \mathrm{x}$ $100)$.

\section{RESULTS AND DISCUSSION}

3.1 Qualitative analysis of the content of active biological substances in fine basil

Cromatograms were viewed in four stages: viewing at 254 $\mathrm{nm}$, before spraying with identification reagent. The purpose of this analysis was to identify the presence of polyphenolic 
antioxidant compound types; viewing at $366 \mathrm{~nm}$ before spraying with identification reagent; viewing at $366 \mathrm{~nm}$ after spraying with identification reagent, when the main types of flavonoids are highlighted in spots by position and size; view after spraying with DPPH reagent in order to identify any antiradical capacity.

Chemical qualitative study conducted by thin layer chromatography showed that, depending on the extraction solvent and extraction conditions (temperature, extraction technique), the obtained extractive solutions have a varied content of phenolic substances (flavonoids and polyphenols), responsible for the antioxidant action of the plants studied.

Comparing chromatoplates' appearance corresponding to $\mathrm{K}$ and $\mathrm{M}$ samples shows the following: - content of flavonoid compounds in the K sample is lower than that in the M sample, although the amount and type of polyphenolcarboxylic acids are comparable; antioxidant activity is attributed mostly to polyphenolcarboxylic acids; extracts obtained with water, respectively alcohol $96^{\circ}$, by stirring at room temperature are significantly lower than those obtained using the same solvent extraction, but at reflux temperature; optimal extraction of active ingredients with antioxidant activity is provided by the mixture of methanol/water (50:50) at reflux temperature.

\subsection{Quantitative analysis of the content of active biological substances in fine basil}

The results of spectrophotometric determinations are summarized in Table 1 . As a first observation we concur that $\mathrm{K}$ sample's hot methanol extraction solution is the most complete. At the opposite pole stands cold ethanol extraction, the value being almost four times less than the maximum. The content of flavonoids in the extracts decreases in the order: methanol, hot> water, hot> methanol-acetone-water-formic acid > ethanol, hot> methanol, cold water-methanol-acetic acid > ethanol at cold water cooling. The $\mathrm{M}$ sample we can observe a different order for lower values.

If one compares the recorded values for flavonoids determination in tinctures (extracts from cold), it appears that they are much lower than extracts made at reflux temperature. This is evidence that flavonoids have increasingly better temperature extractability. Modest results were obtained for complex solvent extractions $\left(\mathrm{S}_{4}\right.$ and $\left.\mathrm{S}_{5}\right)$. Comparing the two samples, the plant-spice has higher values for most types of extractions (six out of eight).

Total polyphenol concentrations are consistent with the literature, with one exception: the ethanol extract, cold (Table 2). It can be noted the wide range of polyphenol content of basil in the literature data (the difference between the maximum and minimum being $5.81 \mathrm{~g} \mathrm{GAE} / 100$ s.u.), because the tests were conducted on different species of plants, some used as spices (mainly due to the contribution of volatile oil), others for medicinal purposes (due to complex bioactive), differing also in how the extraction is carried out.

\begin{tabular}{|c|c|c|c|c|c|c|c|}
\hline \multirow[t]{2}{*}{$\begin{array}{l}\text { Crt } \\
\text { No. }\end{array}$} & \multirow[t]{2}{*}{ Sample } & \multicolumn{2}{|c|}{$\begin{array}{l}\text { Flavonoids } \\
\text { [g rutoside/ } \\
100 \mathrm{~g} \text { s.u.] }\end{array}$} & \multicolumn{2}{|c|}{ 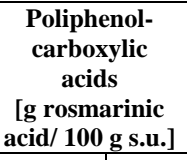 } & \multicolumn{2}{|c|}{$\begin{array}{c}\text { Poliphenols } \\
\text { [g GAE/ } \\
100 \text { g s.u.] }\end{array}$} \\
\hline & & $\mathbf{K}$ & M & $\mathbf{K}$ & M & $\mathbf{K}$ & M \\
\hline 1. & $S_{1 R}$ & 0,10 & 0,20 & 0,06 & 0,40 & 1,46 & 3,91 \\
\hline 2. & $S_{1 C}$ & 0,30 & 0,43 & 0,64 & 0,59 & 5,00 & 4,46 \\
\hline 3. & $\mathrm{~S}_{2 \mathrm{R}}$ & 0,25 & 0,22 & 0,53 & 0,73 & 3,65 & 5,35 \\
\hline 4. & $\mathrm{~S}_{2 \mathrm{C}}$ & 0,46 & 0,55 & 0,81 & 1,24 & 6,39 & 5,01 \\
\hline 5. & $\mathrm{~S}_{3 \mathrm{R}}$ & 0,13 & 0,09 & 0,05 & 0,17 & 0,33 & 1,47 \\
\hline 6. & $S_{3 C}$ & 0,26 & 0,07 & 0,01 & 0,16 & 1,72 & 1,76 \\
\hline 7. & $\mathrm{~S}_{4}$ & 0,25 & 0,14 & 0,10 & 0,34 & 1,30 & 2,98 \\
\hline 8. & $\mathrm{~S}_{5}$ & 0,28 & 0,03 & 0,36 & 0,02 & 2,61 & 1,05 \\
\hline
\end{tabular}

Tab. 1. Quantitative determination of active biological substances in basil extracts

\begin{tabular}{|c|c|c|c|c|}
\hline \multirow{2}{*}{ Sample } & \multicolumn{2}{|c|}{$\begin{array}{c}\text { Polyphenol content of } \\
\text { analyzed samples } \\
\text { [g GAE/ 100 g s.u] }\end{array}$} & \multicolumn{2}{|c|}{$\begin{array}{c}\text { Literature data } \\
\text { [g GAE/ 100 g s.u] }\end{array}$} \\
\cline { 2 - 3 } & Minimum & Maximum & Minimum & Maximum \\
\hline 1 & 0,31 & 5,98 & \multirow{2}{*}{0,74} & 6,55 \\
\hline 2 & 0,98 & 4,68 & & \multirow{2}{*}{} \\
\hline
\end{tabular}

Tab. 2. Comparison of polyphenol content in the analyzed samples with data from literature

The analysis showed that the sample obtained by cold extraction with methanol solution was most effective in removing the DPPH radicals (Table 3). The values obtained in the determination of antioxidant capacity are considerable for many extractions.

\begin{tabular}{|c|c|c|c|}
\hline \multirow{2}{*}{$\begin{array}{c}\text { Crt. } \\
\text { No. }\end{array}$} & \multirow{2}{*}{$\begin{array}{c}\text { Solvent } \\
\text { (sample) }\end{array}$} & \% antioxidant activity against DPPH \\
\cline { 3 - 4 } & $\mathrm{S}_{1 \mathrm{R}}$ & $\mathbf{K}$ & $\mathbf{M}$ \\
\hline 1. & $\mathrm{~S}_{1 \mathrm{C}}$ & 63,55 & 59,95 \\
\hline 2. & $\mathrm{~S}_{2 \mathrm{R}}$ & 98,70 & 89,17 \\
\hline 3. & $\mathrm{~S}_{2 \mathrm{C}}$ & 89,40 & 90,76 \\
\hline 4. & $\mathrm{~S}_{3 \mathrm{R}}$ & 12,01 & 89,27 \\
\hline 5. & $\mathrm{~S}_{3 \mathrm{C}}$ & 53,23 & 14,94 \\
\hline 6. & $\mathrm{~S}_{4}$ & 4,72 & 91,90 \\
\hline 7. & $\mathrm{~S}_{5}$ & 75,35 & 75,10 \\
\hline 8. & & & 8,14 \\
\hline
\end{tabular}

Tab. 3. Antioxidant activity of basil extracts

It can also be noted that high values of antioxidant capacity were obtained for complex solvent extracts, i.e. extracts from cold, although the values obtained for polyphenolic compounds have overwhelmingly modest values.

\section{CONCLUSION}

Making determinations on several types of extracts can give indications about the availability of bioactive compounds in certain environments which differ in temperature, $\mathrm{pH}$, hydro-or lipofil character. The analyzed bioactive compounds are well represented in the basil, but identifying them requires selection of optimal extraction conditions. In general, the principle of determination and analysis technique used influences the results obtained in determining antioxidant activity and compounds with antioxidant potential.

Results of the study point out that aromatic plant have a considerable amount of polyphenolic compounds and a high antiradical activity, while the most effective method of extracting biologically active compounds was the extraction with $50 \%$ methanol solution, hot. In choosing raw materials to obtain spices special attention should be given to their chemical compounds content which leads to a high antioxidant potential. Thus, it is possible to increase dietary intake of antioxidants with beneficial health effects.

\section{REFERENCES}

Cai, Y., et al. (2004). Antioxidant activity and phenolic compounds of 112 traditional Chinese medicinal plants associated with anticancer. Life Science, 74 (17), 2157-2184

Kaefer, C. \& Milner, J. (2008). The role of herbs and spices in cancer prevention, Journal of Nutritional Biochemistry, 19, 347-361

Lu, Mei; Yuan, B.; Zeng, M \& Chen, J. (2010). Antioxidant capacity and major phenolic compounds of spices commonly consumed in China, Food Research International, Available from: http://www.elsevier.com

Shan, B., et al. (2005). Antioxidant capacity of 26 spice extracts and characterization of their phenolic constituents, Journal of Agricultural and Food Chemistry, 53(20), 7749-7759

Suhaj, M. (2006). Spice antioxidants isolation and their antiradical activity: a review. Journal of Food Composition and Analysis, $19,531-537$ 